\title{
DESEMPENHO PRODUTIVO DE GENÓTIPOS DE MILHO SOB DÉFICIT HÍDRICO
}

\author{
RICARDO SILVA DE SOUSA ${ }^{1}$, EDSON ALVES BASTOS², MILTON JOSÉ CARDOSO², \\ VALDENIR QUEIROZ RIBEIRO² e RAMILOS RODRIGUES DE BRITO ${ }^{3}$
}

${ }^{1}$ UFPI, Teresina, PI, Brasil, ricardoss@ufpi.edu.br

${ }^{2}$ Embrapa Meio-Norte,Teresina,PI,Brasil,edson.bastos@embrapa.br,milton.cardoso@embrapa.br,valdenir.queiroz@embrapa.br

${ }^{3}$ Unesp, Botucatu, SP, Brasil, ramilos@hotmail.com

Revista Brasileira de Milho e Sorgo, v.14, n.1, p. 49-60, 2015

RESUMO - O trabalho objetivou avaliar o desempenho produtivo de genótipos de milho sob deficiência hídrica, visando a identificar aqueles com tolerância à seca. Dois experimentos, sem e com déficit hídrico durante a fase reprodutiva, foram conduzidos no campo experimental da Embrapa Meio-Norte, em Teresina, PI, de setembro a dezembro de 2010 em delineamento de blocos ao acaso com 36 tratamentos (genótipos) e quatro repetições. As lâminas de irrigação, somadas à precipitação pluvial, totalizaram $691,6 \mathrm{~mm}$ e 490,8 mm para os experimentos sob irrigação plena (IP) e sob déficit hídrico (DH), respectivamente. A fração de esgotamento de água no solo atingiu valores máximos de $26 \%$ e $75 \%$, respectivamente, para os experimentos IP e DH. Avaliaram-se, em ambos os experimentos, o $\mathrm{n}^{\mathrm{o}}$ de grãos $\left(\mathrm{NG} \mathrm{m}^{-2}\right)$ e o $\mathrm{n}^{\mathrm{o}}$ de espigas por metro quadrado $\left(\mathrm{NE} \mathrm{m}^{-2}\right)$, a produtividade de grãos secos (PGS) com 13\% de umidade e a eficiência do uso da água (EUA). O cultivo sob déficit hídrico no solo apresentou $59,23 \%$ de redução no $\mathrm{NG} \mathrm{m}^{-2} ; 33,27 \%$ no $\mathrm{NE} \mathrm{m}^{-2} ; 59,5 \%$ na PGS; e 42,9\% na EUA. Identificaram-se os genótipos com melhor desempenho sob déficit hídrico: AG 7088; 3G 733 5; 1G 703 4; 3G 739 5; 3G 741 5; 2H 828; e 3H 843.

Palavras-Chave: Rendimento de grãos; eficiência de uso da água; componentes de produção.

\section{PERFORMANCE OF MAIZE GENOTYPES UNDER WATER DEFICIT}

\begin{abstract}
The work aimed to evaluate the performance of maize genotypes under water deficit, in order to identify those with drought tolerance. Two experiments, with and without water deficit during the reproductive phase, were conducted in the experimental area of Embrapa Meio-Norte in Teresina, PI, in the period from September to December 2010, in a randomized block design with 36 treatments (genotypes) and four replications. The sum of irrigation depth and rainfall totaled $691.6 \mathrm{~mm}$ and $490.8 \mathrm{~mm}$ for the experiments under full irrigation (FI) and water deficit, (WD), respectively. The fraction of soil water depletion reached maximum values of 26 and $75 \%$ for FI and $\mathrm{WD}$, respectively. The traits evaluated in both experiments were: number of grains $\left(\mathrm{NG} \mathrm{m}^{-2}\right)$, number of ears per square meter $\left(\mathrm{NE} \mathrm{m}^{-2}\right)$, grain yield (GY) with 13\% humidity, and the water use efficiency (WUE). Cultivation under

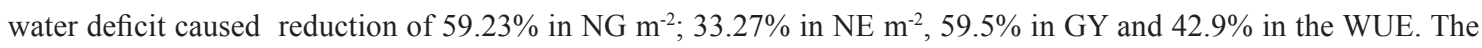
genotypes with the best performance under water deficit were: AG 7088, 3G 733 5, 1G 703 4, 3G 739 5, $3 \mathrm{G} 7415$, $2 \mathrm{H} 828$ and $3 \mathrm{H} 843$.
\end{abstract}

Key words: Grain yield; water use efficiency; yield components. 
O milho (Zea mays L.) é o cereal mais produzido no mundo. No Nordeste brasileiro, uma das maiores regiões produtoras de grãos, registrou-se, em cultivo de sequeiro, uma produção de 5.138.600 t em uma área de 2.407.500 ha na safra de 2012/2013 (Conab, 2013). Considerando o seu potencial produtivo, o rendimento médio de grãos na região Nordeste brasileira ainda é baixo, apenas $2.134 \mathrm{~kg} \mathrm{ha}^{-1}$. Dentre os fatores que comprometem a produção e contribuem para o baixo rendimento, encontram-se a ausência de genótipos mais tolerantes ao déficit hídrico e a não uniformidade das precipitações, com a ocorrência de veranicos muitas vezes prolongados na fase crítica de desenvolvimento da cultura.

O déficit hídrico na planta, dependendo de sua intensidade e da fase do ciclo da cultura, pode reduzir severamente o rendimento da cultura. Para Allen et al. (1998), a cultura começa a entrar em déficit hídrico quando o conteúdo de água no solo passa de um valor limite, em que a água não pode ser transportada rapidamente por meio das raízes para responder à demanda transpirométrica. De acordo com Doorenbos \& Kassam (1994); Bruce et al. (2002) e Bai et al. (2006), o efeito do déficit hídrico sobre o crescimento e o rendimento de uma cultura depende da espécie, da variedade e da duração e da intensidade do déficit hídrico. Bergamaschi et al. (2004) ressaltam que o milho, na fase do pendoamento até o início do enchimento de grãos, é extremamente sensível ao déficit hídrico.

Diversas características que ajudam a identificar genótipos tolerantes à deficiência hídrica são avaliadas, dentre essas o rendimento de grãos, a eficiência de uso da água, além dos componentes de produção (número de grãos por metro quadrado e número de espigas por metro quadrado), que são correlacionados positiva ou negativamente com o rendimento da cultura (Bergamaschi et al., 2004; Cardoso et al., 2010; Gomide et al., 2011; Bastos et al., 2012). Para Bergamaschi et al. (2004), os componentes de produção mais afetados pelo déficit hídrico são o número de grãos por espiga e o número de espigas por planta, tendo em vista que esses são definidos no período crítico da cultura e, portanto, sofrem efeitos mais intensos nesse momento.

Trabalhos como o de Çakir (2004) e Beiragi et al. (2011) revelaram que os componentes de produção e o rendimento de grãos de milho foram significativamente afetados pelo déficit hídrico. Beiragi et al. (2011) observaram redução média para rendimento de grãos de $71,54 \%$, quando a fração de esgotamento de água no solo atingiu $80 \%$.

Para Doorenbos \& Kassan (1994), quando o suprimento de água não atende às necessidades hídricas da cultura, essas variam em suas respostas aos déficits hídricos. Em algumas culturas, há um aumento na eficiência de utilização de água, enquanto em outras há uma diminuição, com o aumento do déficit hídrico. Para Magalhães et al. (2009), as linhagens tolerantes à deficiência hídrica apresentaram maior eficiência no uso da água.

Nesse sentido, acredita-se que a melhor alternativa da produção agrícola, buscando melhor rendimento e estabilidade de produção sob condição de déficit hídrico, será a identificação de variedades tolerantes a esse estresse ambiental. Diante do exposto, o trabalho teve como objetivo avaliar o efeito do déficit hídrico sobre o rendimento de grãos; componentes de produção e eficiência do uso da água, bem como identificar genótipos de milho com características de tolerância ao déficit hídrico. 


\section{Material e Métodos}

Foram conduzidos dois experimentos com a cultura do milho, um sob deficiência hídrica (DH) e outro sob irrigação plena (IP), no campo experimental da Embrapa Meio-Norte, em Teresina, PI $\left(05^{\circ} 05^{\prime}\right.$ S; 42 $48^{\prime}$ ' W e 74,4m), de setembro a dezembro de 2010. O experimento IP foi utilizado como referência, pois adotou-se como critério para identificação de genótipos com tolerância à seca os que produzissem acima da média entre todos os genótipos (na condição de déficit hídrico) e cuja redução de produtividade de grãos em relação ao regime de irrigação plena fosse inferior ou igual a $50 \%$, tendo em vista que a maioria dos cereais sob déficit moderado reduz sua produção em não mais que cerca de 50\% em comparação com aqueles em condição de não déficit (Araus et al., 2008).

De acordo com Bastos \& Andrade Júnior (2008), considerando a série histórica de 1980 a 2007, as médias anuais de temperatura do ar, de umidade relativa do ar e de precipitação são de $28,2{ }^{\circ} \mathrm{C}$, $72,6 \%$ e $1.336 \mathrm{~mm}$, respectivamente, concentrando a maioria das chuvas nos meses de janeiro a abril. Os valores médios mensais de temperatura máxima, média e mínima do ar no decorrer dos experimentos foram $36,6{ }^{\circ} \mathrm{C}, 29,3{ }^{\circ} \mathrm{C}$ e $23,1{ }^{\circ} \mathrm{C}$, respectivamente, com umidade relativa média de $62 \%$ e precipitação pluvial de 259,6 $\mathrm{mm}$.

O solo da área experimental é um argissolo amarelo distrófico de textura franco arenosa. A análise granulométrica do solo, considerando-se a camada de 0-40 cm, apresentou em média 764,5 $\mathrm{g} \mathrm{kg}^{-1}$ de areia, $104,5 \mathrm{~g} \mathrm{~kg}^{-1}$ de silte e $131 \mathrm{~g} \mathrm{~kg}^{-1}$ de argila. O valor da capacidade de campo, CC (10 kPa), considerando a camada de 0 a $40 \mathrm{~cm}$, é de $21 \%$ e o ponto de murcha permanente, PMP (1.500 kPa), corresponde a 9\% a base de volume determinados em câmara de pressão de Richards, conforme descrito em Embrapa (1997). A adubação em fundação constou da aplicação de 40 $\mathrm{kg}$ de $\mathrm{N} \mathrm{ha}^{-1}, 80 \mathrm{~kg}$ de $\mathrm{P}_{2} \mathrm{O}_{5}$ ha $^{-1}$ e $35 \mathrm{~kg}$ de $\mathrm{K}_{2} \mathrm{O}$ ha $^{-1}$. A adubação de cobertura foi parcelada em duas aplicações, sendo a primeira na ocasião da sexta folha, com $40 \mathrm{~kg}$ de $\mathrm{N} \mathrm{ha}^{-1}$ e $35 \mathrm{~kg}$ de $\mathrm{K}_{2} \mathrm{O} \mathrm{ha}^{-1}$, e a segunda na emissão da oitava folha, com $40 \mathrm{~kg}$ de $\mathrm{N} \mathrm{ha}^{-1}$.

$\mathrm{O}$ delineamento utilizado nos experimentos foi em blocos casualizados com quatro repetições, parcelas experimentais constituídas de uma fileira de $4 \mathrm{~m}$ de comprimento, espaçadas $0,80 \mathrm{~m}$ entre si e com 0,20 m entre covas, totalizando 144 parcelas, com área útil de 3,2 $\mathrm{m}^{2}$ em cada experimento (IP e DH). Os tratamentos consistiram de 36 híbridos de milho (Tabela 1) procedentes da Embrapa Milho e Sorgo, Sete Lagoas, MG.

A área experimental foi irrigada utilizando um sistema de irrigação por aspersão convencional com aspersores espaçados $12 \mathrm{~m}$ x $12 \mathrm{~m}$, com vazão de $1,6 \mathrm{~m}^{3} \mathrm{~h}^{-1}$. As lâminas de irrigação aplicadas foram medidas em baterias de 16 coletores instalados em cada experimento que, somadas à precipitação pluvial, totalizaram 691,6 mm e 490,8 mm, respectivamente, para IP e DH. O manejo de irrigação foi realizado com base na evapotranspiração da cultura (ETc), que foi calculada por meio do coeficiente de cultura (Kc) proposto por Andrade Júnior et al. (1998), e da evapotranspiração de referência (ETo), estimada pelo método de Penman-Monteith por meio dos dados climáticos obtidos de uma estação meteorológica da Embrapa Meio-Norte, situada aproximadamente a $400 \mathrm{~m}$ da área experimental.

O monitoramento do teor de água no solo foi realizado diariamente por meio de uma sonda de capacitância, modelo DIVINER $2000^{\circledR}$, em camadas de $0,10 \mathrm{~m}$, até $0,70 \mathrm{~m}$ de profundidade, a partir 
de 17 dias após a semeadura (DAS) até o final do ciclo da cultura. Os dados de umidade foram convertidos para lâmina de armazenamento de água e comparados com dados de disponibilidade de água no solo. A reposição foi realizada quando a fração de esgotamento de água no solo atingiu o valor de 26\%. No experimento sob deficiência hídrica, a irrigação foi interrompida do estádio VT (pendoamento) ao R2 (grão leitoso) da cultura, período entre o $50^{\circ}$ e $64^{\circ}$ DAS.

A produtividade de grãos secos (PGS) foi obtida a partir das espigas colhidas na área útil da parcela que, em seguida, foi transformada em $\mathrm{kg} \mathrm{ha}^{-1}$, corrigida para $13 \%$ de umidade. A eficiência do uso da água (EUA) foi determinada pela equação:

$$
E U A=\frac{P G S}{L a p}
$$

em que Lap é lâmina de água aplicada.

$\mathrm{O}$ número de grãos por metro quadrado $(\mathrm{NG}$ $\mathrm{m}^{-2}$ ) foi encontrado pela equação:

$$
N G \cdot m^{2}=\frac{P G \cdot m^{2}}{P 100 G}
$$

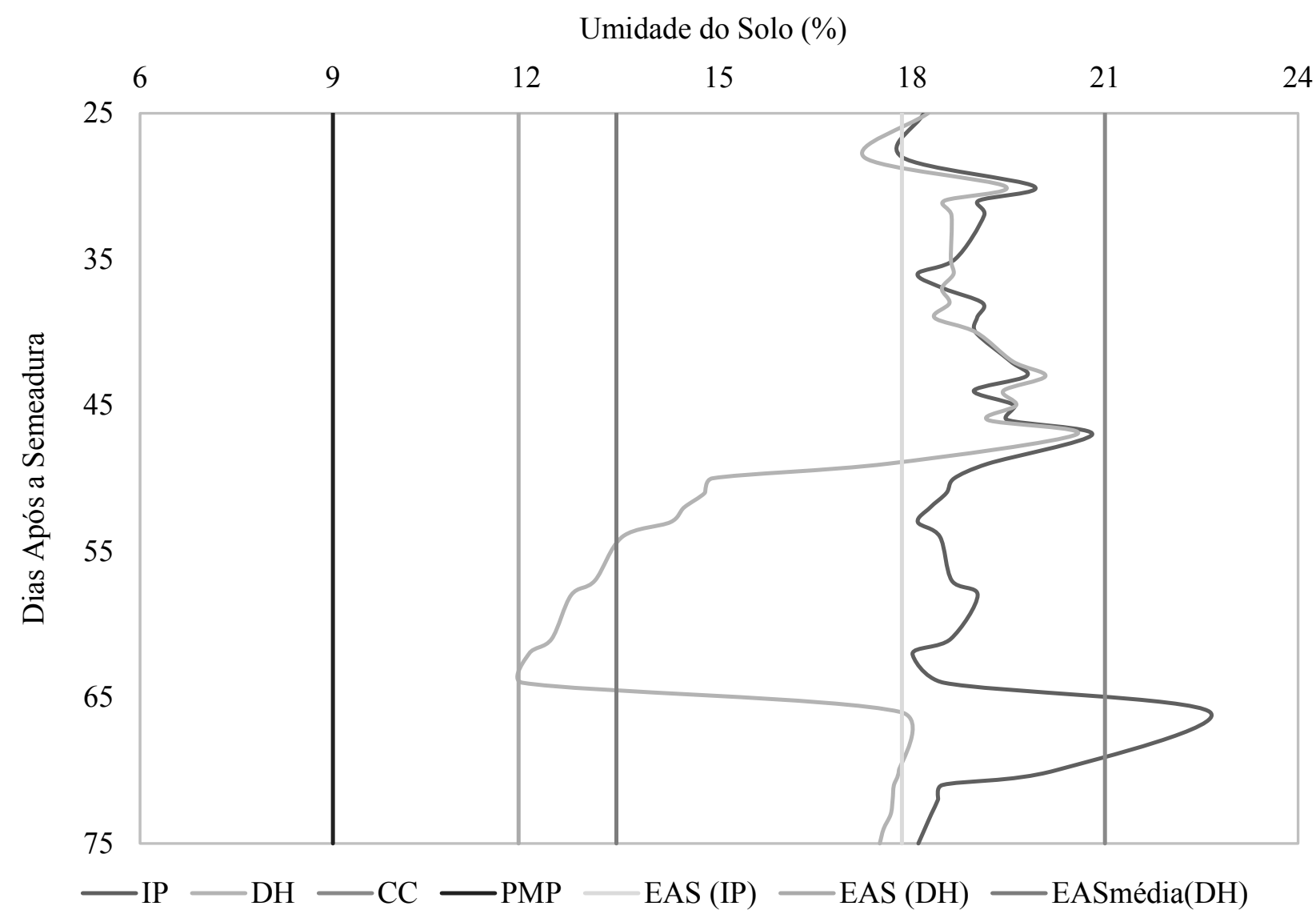

FIGURA 1. Variação da umidade do solo (\%) até uma profundidade de $0,40 \mathrm{~m}$ ao longo do ciclo fenológico de genótipos de milho submetidos às condições de irrigação plena (IP) e deficiência hídrica (DH) observados a capacidade de campo (CC), o ponto de murcha permanente (PMP) e o esgotamento de água no solo (EAS). Teresina, PI, 2010. 
em que $P G \mathrm{~m}^{-2}$ é o peso de grãos por metro quadrado; $P 100 G$ é o peso de 100 grãos.

$\mathrm{O}$ número de espigas por metro quadrado $\left(\mathrm{NE} \mathrm{m}^{-2}\right)$ foi determinado pela equação:

$$
N E \cdot m^{2}=\frac{N E p}{A p}
$$

em que $N E p$ é o número de espigas na parcela; $A p$ é a área útil da parcela.

Os dados sobre a PGS $\left(\mathrm{kg} \mathrm{ha}^{-1}\right)$ e a EUA ( $\mathrm{kg}$. $\mathrm{ha}^{-1} \mathrm{~mm}^{-1}$ ) foram submetidos à análise de variância, depois de verificada homogeneidade de acordo com as metodologias de Pimentel-Gomes (1990) e Zimmermann (2004), sendo a comparação entre os tratamentos realizada pelo teste de Scott-Knott a 5\% de probabilidade (Cruz, 2006). Para a análise estatística dos componentes de produção $\left(\mathrm{NG} \mathrm{m}^{-2} \mathrm{e} \mathrm{NE} \mathrm{m}^{-2}\right)$, utilizou-se transformação dos dados originais para . Todas as análises de variâncias foram realizadas usando o programa SAS (SAS Institute Inc., 1989).

\section{Resultados e Discussão}

O monitoramento da umidade do solo indicou que, no experimento sob irrigação plena, os valores mínimos de tensão alcançaram $-26 \mathrm{kPa}$, o que equivale a $18 \%$ de umidade no solo e corresponde a $26 \%$ de esgotamento de água no solo (EAS). Por outro lado, sob déficit hídrico, a tensão crítica alcançou $-290 \mathrm{kPa}$, equivalendo a $12 \%$ de umidade e a uma fração de $75 \%$ de EAS. Considerando que Doorembos e Kassam (1994) recomendam uma fração de $50 \%$ de esgotamento de água no solo para a cultura do milho e as produtividades de grãos obtidas neste trabalho (de $1.404,9$ a $8.413,3 \mathrm{Kg}$ $\mathrm{ha}^{-1}$ ) (Tabela 1), pode-se inferir que ocorreu déficit hídrico moderado.
O déficit hídrico influenciou na produtividade de grãos secos (PGS), nos componentes de produção e na eficiência do uso da água (EUA) e revelou diferença significativa entre os genótipos. Observou-se que a PGS (Tabela 1) variou significativamente entre os genótipos, nas duas condições hídricas, possivelmente em virtude da variabilidade genética entre esses, haja vista que o rendimento de grãos é função do potencial da planta para reagir às condições sob as quais a mesma está se desenvolvendo (Ritchie et al., 1993). Sete genótipos se destacaram quanto aos critérios de identificação para a tolerância à deficiência hídrica, AG 7088, 3G 733 5, $1 \mathrm{G} 703$ 4, 3G 739 5, 3G 741 5, $2 \mathrm{H} 828$ e $3 \mathrm{H}$ 843. No ensaio sob irrigação plena, a PGS variou de 5.332,79 $\mathrm{kg} \mathrm{ha}^{-1}(3 \mathrm{H} 813)$ a $11.018,00$ $\mathrm{kg} \mathrm{ha}^{-1}$ (AG 7088), enquanto sob deficiência hídrica de $1.404,92 \mathrm{~kg} \mathrm{ha}^{-1}$ (1F 625 5) a 8.413,32 $\mathrm{kg} \mathrm{ha}^{-1}$ (AG 7088), quando foi observada uma redução média de $59,5 \%$, considerando o experimento sob irrigação plena como referência. Essa redução é menor que a encontrada (71,8\%) por Bastos et al. (2012), quando objetivaram identificar genótipos de milho com tolerância à deficiência hídrica e com maior eficiência no uso da água, tendo em vista que os referidos autores utilizaram déficit moderado.

Quanto aos valores médios do número de grãos por metro quadrado $\left(\mathrm{NG} \mathrm{m}^{-2}\right)$ e do número de

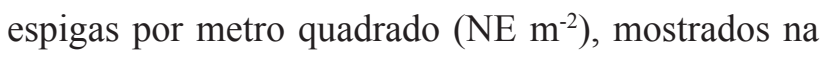
Tabela 2, foram observadas reduções médias de 59,23 $\%$ e 33,27 \%, respectivamente, sob condição de déficit hídrico em relação ao experimento com irrigação plena. Reduções desses componentes também foram observadas por Cardoso et al. (2011) em genótipos de milho sob condição de déficit hídrico. A redução no $\mathrm{NG} \mathrm{m}^{-2}$ pode ser atribuída à quebra de sincronismo entre o pendoamento, que geralmente ocorre mais cedo, e o espigamento, que ocorre mais tarde. Tal fato 
TABELA 1. Valores e redução médios da produtividade de grãos secos (PGS) de genótipos de milho submetidos a condições de irrigação plena (IP) e deficiência hídrica (DH) do estádio VT ao R2. Teresina, PI, 2010.

\begin{tabular}{|c|c|c|c|}
\hline \multirow{2}{*}{ Genótipo $^{1}$} & \multicolumn{2}{|c|}{ PGS $\left(\mathrm{kg} \mathrm{ha}^{-1}\right)$} & \multirow{2}{*}{ Redução (\%) } \\
\hline & DH & IP & \\
\hline AG 7088 & $8413,32 \mathrm{~A}$ & $11018,00 \mathrm{~A}$ & 23,64 \\
\hline $3 G 7335$ & 4834,46 B & $6651,87 \mathrm{E}$ & 27,32 \\
\hline $1 G 7034$ & $4710,40 \mathrm{~B}$ & $8508,32 \mathrm{C}$ & 44,64 \\
\hline $3 \mathrm{G} 7395$ & $4361,40 \mathrm{~B}$ & $6701,82 \mathrm{E}$ & 34,92 \\
\hline $3 \mathrm{G} 7415$ & $4157,07 \mathrm{~B}$ & $7127,82 \mathrm{D}$ & 41,68 \\
\hline $2 \mathrm{H} 828$ & $3761,17 \mathrm{C}$ & $5430,53 \mathrm{E}$ & 30,74 \\
\hline $3 \mathrm{H} 843$ & $3752,90 \mathrm{C}$ & $6387,50 \mathrm{E}$ & 41,25 \\
\hline $2 \mathrm{H} 834$ & $3532,42 \mathrm{C}$ & 9280,97 B & 61,94 \\
\hline 1H 795 & $3492,35 \mathrm{C}$ & $8243,90 \mathrm{C}$ & 57,64 \\
\hline 1D 2255 & $3402,95 \mathrm{C}$ & $8336,22 \mathrm{C}$ & 59,18 \\
\hline 1F 5834 & $3351,85 \mathrm{C}$ & $6393,79 \mathrm{E}$ & 47,58 \\
\hline 1F 5574 & $3270,40 \mathrm{C}$ & $7434,55 \mathrm{D}$ & 56,01 \\
\hline $1 F 6325$ & $3052,60 \mathrm{D}$ & $8199,77 \mathrm{C}$ & 62,77 \\
\hline $1 \mathrm{G} 6724$ & $3042,62 \mathrm{D}$ & $7325,75 \mathrm{D}$ & 58,47 \\
\hline $3 F 6245$ & $3015,17 \mathrm{D}$ & $8273,80 \mathrm{C}$ & 63,56 \\
\hline $3 \mathrm{H} 823$ & $3008,85 \mathrm{D}$ & $7123,67 \mathrm{D}$ & 57,76 \\
\hline $1 \mathrm{H} 845$ & $2984,30 \mathrm{D}$ & $6348,95 \mathrm{E}$ & 53,00 \\
\hline 3Н 832 & $2962,90 \mathrm{D}$ & $6819,00 \mathrm{D}$ & 56,55 \\
\hline 3Н 798 & $2883,07 \mathrm{D}$ & $6150,52 \mathrm{E}$ & 53,12 \\
\hline 2B 707 & $2794,47 \mathrm{D}$ & $7471,85 \mathrm{D}$ & 62,60 \\
\hline $2 \mathrm{H} 829$ & $2707,57 \mathrm{D}$ & $7587,10 \mathrm{D}$ & 64,31 \\
\hline $3 \mathrm{H} 813$ & $2414,12 \mathrm{E}$ & $5332,79 \mathrm{E}$ & 54,73 \\
\hline $3 \mathrm{G} 7385$ & $2354,32 \mathrm{E}$ & $8402,17 \mathrm{C}$ & 71,98 \\
\hline $3 \mathrm{H} 842$ & $2260,35 \mathrm{E}$ & $7748,20 \mathrm{C}$ & 70,83 \\
\hline $3 \mathrm{E} 4824$ & $2256,82 \mathrm{E}$ & $7496,65 \mathrm{D}$ & 69,90 \\
\hline $2 \mathrm{H} 826$ & $2188,07 \mathrm{E}$ & $7065,10 \mathrm{D}$ & 69,03 \\
\hline
\end{tabular}




\begin{tabular}{cccc} 
2H 831 & $2069,55 \mathrm{E}$ & $6356,57 \mathrm{E}$ & 67,44 \\
30 F35 & $2066,10 \mathrm{E}$ & $9843,87 \mathrm{~B}$ & 79,01 \\
2E 5305 & $2040,35 \mathrm{E}$ & $7987,55 \mathrm{C}$ & 74,46 \\
1F 62 65 & $1992,17 \mathrm{E}$ & $6597,07 \mathrm{E}$ & 69,80 \\
BRS 1040 & $1956,47 \mathrm{E}$ & $5867,82 \mathrm{E}$ & 66,66 \\
3F 627 5 & $1913,45 \mathrm{E}$ & $7694,05 \mathrm{C}$ & 75,13 \\
1D 219 5 & $1836,55 \mathrm{E}$ & $6167,95 \mathrm{E}$ & 70,22 \\
1F 592 4 & $1571,72 \mathrm{~F}$ & $6709,30 \mathrm{E}$ & 76,57 \\
1H 787 & $1413,17 \mathrm{~F}$ & $8283,37 \mathrm{C}$ & 82,94 \\
1F 625 5 & $1404,92 \mathrm{~F}$ & $6390,75 \mathrm{E}$ & 78,02 \\
\hline Média & 2978,60 & 7354,40 & 59,50 \\
CV (\%) & 14,33 & 10,52 & --- \\
Teste F & $* *$ & $* *$ & --- \\
\hline
\end{tabular}

${ }^{1}$ Médias seguidas da mesma letra, maiúscula na coluna, não diferem entre si pelo teste de Scott-Knott a 5\% de probabilidade; ${ }^{* *}$ Significativo ao nível de $1 \%$ de probabilidade pelo teste $\mathrm{F}$.

faz com que haja abortamento dos grãos de pólen ou que, no momento da emissão dos estilos estigmas, os grãos de pólen não estejam mais viáveis, resultando, assim, em espigas com poucos grãos ou sem grãos. A redução no $\mathrm{NE} \mathrm{m}^{-2}$, de acordo com Cardoso et al. (2010), também pode ser atribuída ao atraso provocado pelo déficit hídrico na emissão da mesma, o que levou muitas plantas a perderem sua capacidade de emissão de espigas por causa do estado debilitado em que se encontravam ao final da restrição hídrica.

Esses componentes, $\mathrm{NG} \mathrm{m}^{-2}$ e $\mathrm{NE} \mathrm{m}^{-2}$, revelaram possuir forte grau de correlação positiva $(0,95) \mathrm{e}$ $(0,65)$, respectivamente, com a PGS indicando serem importantes para predição da PGS sob condição de deficiência hídrica na fase crítica da cultura, haja vista serem mais afetados nesse momento.

O déficit hídrico teve influência significativa na eficiência do uso da água (Tabela 3), cujos valores médios foram de 10,63 e $6,07 \mathrm{~kg} \mathrm{ha}^{-1} \mathrm{~mm}^{-1}$ para condição de irrigação plena e sob deficiência hídrica, respectivamente. Verificou-se que o genótipo AG 7088 apresentou a maior EUA diferindo, estatisticamente, dos demais. Em média, houve aumento de $42,90 \%$ na EUA de genótipos submetidos a déficit hídrico, sendo considerada a condição de irrigação plena como referência. Estes resultados foram inferiores aos obtidos (60,40 \%) por Bastos et al. (2012), quando objetivaram identificar genótipos de milho com tolerância à deficiência hídrica e com maior eficiência no uso da água. Para Magalhães et al. (2009), as linhagens tolerantes à deficiência hídrica apresentam maior eficiência no uso da água. $\mathrm{O}$ aumento da eficiência de uso da água pelos híbridos pode estar associado ao fechamento dos estômatos, pois, segundo Taiz \& Zeiger (2009), o fechamento estomático inibe a transpiração mais do que diminui as concentrações intercelulares de $\mathrm{CO}_{2}$. Entretanto, é desejável que genótipos com tolerância à deficiência hídrica utilizem 
TABELA 2. Valores médios do $\mathrm{n}^{0}$ de grãos por metro quadrado $\left(\mathrm{NG} \mathrm{m}^{-2}\right)$ e do $\mathrm{n}^{\mathrm{o}}$ de espigas por metro quadrado $\left(\mathrm{NE} \mathrm{m}^{-2}\right)$ de genótipos de milho submetidos a condições de irrigação plena (IP) e deficiência hídrica (DH) do estádio VT ao R2. Teresina, PI, 2010.

\begin{tabular}{|c|c|c|c|c|c|c|c|c|}
\hline \multirow{3}{*}{$\begin{array}{l}\text { Genótipo }^{1} \\
\text { AG } 7088\end{array}$} & \multicolumn{4}{|c|}{$\mathrm{NG} \mathrm{m}^{-2}$} & \multicolumn{4}{|c|}{$\mathrm{NE} \mathrm{m}^{-2}$} \\
\hline & \multicolumn{2}{|c|}{ DH } & \multicolumn{2}{|c|}{ IP } & \multicolumn{2}{|c|}{ DH } & \multicolumn{2}{|c|}{ IP } \\
\hline & 2757,30 & $52,51^{*} \mathrm{~A}$ & 3911,25 & $62,54^{*} \mathrm{~A}$ & 5,48 & $2,34^{*} \mathrm{~A}$ & 7,18 & $2,68^{*} \mathrm{~A}$ \\
\hline $3 F \quad 6245$ & 919,30 & $30,32 \mathrm{C}$ & 2635,80 & $51,34 \mathrm{~B}$ & 4,75 & $2,18 \mathrm{~B}$ & 6,45 & $2,54 \mathrm{~A}$ \\
\hline 1F 6265 & 701,19 & $26,48 \mathrm{D}$ & 2104,97 & $45,88 \mathrm{C}$ & 3,13 & 1,77 D & 5,76 & $2,40 \mathrm{~B}$ \\
\hline 1H 787 & 464,83 & $21,56 \mathrm{E}$ & 2634,77 & $51,33 \mathrm{~B}$ & 3,13 & 1,77 D & 4,37 & $2,09 \mathrm{C}$ \\
\hline 1D 2195 & 582,74 & $24,14 \mathrm{E}$ & 1966,04 & $44,34 \mathrm{D}$ & 3,10 & $1,76 \mathrm{D}$ & 5,20 & $2,28 \mathrm{C}$ \\
\hline 1D 2255 & 1081,75 & $32,89 \mathrm{C}$ & 2430,49 & $49,30 \mathrm{C}$ & 4,54 & $2,13 \mathrm{~B}$ & 6,10 & $2,47 \mathrm{~B}$ \\
\hline $3 \mathrm{H} 842$ & 729,54 & $27,01 \mathrm{D}$ & 2344,50 & $48,42 \mathrm{C}$ & 2,72 & $1,65 \mathrm{E}$ & 6,86 & $2,62 \mathrm{~A}$ \\
\hline 30 F35 & 589,52 & $24,28 \mathrm{E}$ & 3015,11 & $54,91 \mathrm{~B}$ & 2,34 & $1,53 \mathrm{E}$ & 5,76 & $2,40 \mathrm{~B}$ \\
\hline 1F 5924 & 446,05 & $21,12 \mathrm{E}$ & 2197,73 & $46,88 \mathrm{C}$ & 3,10 & $1,76 \mathrm{D}$ & 4,88 & $2,21 \mathrm{C}$ \\
\hline 2H 834 & 1058,20 & $32,53 \mathrm{C}$ & 2820,67 & $53,11 \mathrm{~B}$ & 3,42 & $1,85 \mathrm{D}$ & 6,86 & $2,62 \mathrm{~A}$ \\
\hline 2E 5305 & 986,59 & $31,41 \mathrm{C}$ & 2661,53 & $51,59 \mathrm{~B}$ & 3,65 & $1,91 \mathrm{D}$ & 6,55 & $2,56 \mathrm{~A}$ \\
\hline 1F 5834 & 1005,52 & $31,71 \mathrm{C}$ & 2326,13 & $48,23 \mathrm{C}$ & 4,20 & $2,05 \mathrm{C}$ & 5,52 & $2,35 \mathrm{C}$ \\
\hline $3 \mathrm{E} 4824$ & 771,17 & $27,77 \mathrm{D}$ & 2408,85 & $49,08 \mathrm{C}$ & 3,10 & $1,76 \mathrm{D}$ & 6,97 & $2,64 \mathrm{~A}$ \\
\hline $3 \mathrm{H} 823$ & 938,81 & $30,64 \mathrm{C}$ & 2258,15 & $47,52 \mathrm{C}$ & 3,88 & $1,97 \mathrm{C}$ & 5,86 & $2,42 \mathrm{~B}$ \\
\hline 2H 826 & 785,68 & $28,03 \mathrm{D}$ & 2202,42 & $46,93 \mathrm{C}$ & 3,65 & $1,91 \mathrm{D}$ & 4,93 & $2,22 \mathrm{C}$ \\
\hline $3 \mathrm{H} 843$ & 1290,96 & $35,93 \mathrm{~B}$ & 1914,94 & $43,76 \mathrm{D}$ & 3,42 & $1,85 \mathrm{D}$ & 4,84 & $2,20 \mathrm{C}$ \\
\hline 2B 707 & 974,06 & $31,21 \mathrm{C}$ & 2589,79 & $50,89 \mathrm{~B}$ & 3,28 & $1,81 \mathrm{D}$ & 5,15 & $2,27 \mathrm{C}$ \\
\hline $2 \mathrm{H} 828$ & 1070,60 & $32,72 \mathrm{C}$ & 1750,59 & $41,84 \mathrm{D}$ & 4,20 & $2,05 \mathrm{C}$ & 5,71 & $2,39 \mathrm{~B}$ \\
\hline 1F 6255 & 428,08 & $20,69 \mathrm{E}$ & 1894,86 & $43,53 \mathrm{D}$ & 3,03 & 1,74 D & 5,20 & $2,28 \mathrm{C}$ \\
\hline $3 F 6275$ & 523,49 & $22,88 \mathrm{E}$ & 2326,13 & $48,23 \mathrm{C}$ & 3,03 & 1,74 D & 5,66 & $2,38 \mathrm{~B}$ \\
\hline 2Н 831 & 686,44 & $26,20 \mathrm{D}$ & 1957,18 & $44,24 \mathrm{D}$ & 4,12 & $2,03 \mathrm{C}$ & 4,84 & $2,20 \mathrm{C}$ \\
\hline $3 \mathrm{G} 7395$ & 1379,38 & $37,14 \mathrm{~B}$ & 2075,71 & $45,56 \mathrm{D}$ & 3,72 & $1,93 \mathrm{D}$ & 5,38 & $2,32 \mathrm{C}$ \\
\hline $3 \mathrm{H} 832$ & 987,84 & $31,43 \mathrm{C}$ & 2007,94 & $44,81 \mathrm{D}$ & 3,57 & 1,89 D & 4,97 & $2,23 \mathrm{C}$ \\
\hline $3 \mathrm{G} 7415$ & 1164,17 & $34,12 \mathrm{C}$ & 2172,49 & $46,61 \mathrm{C}$ & 4,58 & $2,14 \mathrm{~B}$ & 5,20 & $2,28 \mathrm{C}$ \\
\hline $1 \mathrm{G} 7034$ & 1349,83 & $36,74 \mathrm{~B}$ & 2674,96 & $51,72 \mathrm{~B}$ & 4,49 & $2,12 \mathrm{~B}$ & 5,95 & $2,44 \mathrm{~B}$ \\
\hline $3 \mathrm{G} 7335$ & 1481,48 & 38,49 B & 2014,21 & $44,88 \mathrm{D}$ & 4,49 & $2,12 \mathrm{~B}$ & 4,71 & $2,17 \mathrm{C}$ \\
\hline $3 G 7385$ & 713,96 & $26,72 \mathrm{D}$ & 2558,34 & $50,58 \mathrm{~B}$ & 4,04 & $2,01 \mathrm{C}$ & 6,25 & $2,50 \mathrm{~B}$ \\
\hline
\end{tabular}




\begin{tabular}{|c|c|c|c|c|c|c|c|c|}
\hline 1F 5574 & 1094,95 & $33,09 \mathrm{C}$ & 2254,35 & $47,48 \mathrm{C}$ & 3,72 & $1,93 \mathrm{D}$ & 5,62 & $2,37 \mathrm{C}$ \\
\hline 1F 6325 & 894,01 & $29,90 \mathrm{C}$ & 2652,25 & $51,50 \mathrm{~B}$ & 3,80 & $1,95 \mathrm{C}$ & 5,48 & $2,34 \mathrm{C}$ \\
\hline 1H 795 & 972,19 & $31,18 \mathrm{C}$ & 2280,06 & $47,75 \mathrm{C}$ & 3,96 & $1,99 \mathrm{C}$ & 6,05 & $2,46 \mathrm{~B}$ \\
\hline BRS 1040 & 643,64 & $25,37 \mathrm{D}$ & 1892,25 & $43,50 \mathrm{D}$ & 2,16 & $1,47 \mathrm{E}$ & 5,15 & $2,27 \mathrm{C}$ \\
\hline $3 \mathrm{H} 813$ & 732,78 & $27,07 \mathrm{D}$ & 1692,50 & $41,14 \mathrm{D}$ & 3,50 & $1,87 \mathrm{D}$ & 4,71 & $2,17 \mathrm{C}$ \\
\hline $2 \mathrm{H} 829$ & 836,37 & $28,92 \mathrm{D}$ & 2257,20 & $47,51 \mathrm{C}$ & 4,37 & $2,09 \mathrm{C}$ & 5,15 & $2,27 \mathrm{C}$ \\
\hline 3Н 798 & 876,16 & $29,60 \mathrm{C}$ & 1897,47 & $43,56 \mathrm{D}$ & 3,50 & $1,87 \mathrm{D}$ & 5,06 & $2,25 \mathrm{C}$ \\
\hline $1 \mathrm{G} 6724$ & 966,59 & $31,09 \mathrm{C}$ & 2072,07 & $45,52 \mathrm{D}$ & 4,54 & $2,13 \mathrm{~B}$ & 5,95 & $2,44 \mathrm{~B}$ \\
\hline 1H 845 & 983,45 & $31,36 \mathrm{C}$ & 2213,70 & $47,05 \mathrm{C}$ & 5,29 & $2,30 \mathrm{~A}$ & 5,95 & $2,44 \mathrm{~B}$ \\
\hline Média & 940,80 & 30,12 & 2307,43 & 47,86 & 3,75 & 1,93 & 5,62 & 2,37 \\
\hline CV $(\%)$ & & 8,37 & & 5,82 & & 6,86 & & 5,35 \\
\hline Teste F & & $* *$ & & $* *$ & & $* *$ & & ** \\
\hline
\end{tabular}

a água com eficiência tanto na condição de déficit hídrico, como na condição em que as necessidades hídricas estão sendo supridas, tendo em vista que o produtor sempre busca maior rendimento.

\section{Conclusões}

O déficit hídrico reduz o número de grãos por $\mathrm{m}^{-2}$, o número de espigas por $\mathrm{m}^{-2} \mathrm{e}$ o rendimento de grãos e aumenta a eficiência de uso da água de híbridos de milho.

Os híbridos de milho AG 7088, 3G 733 5, $1 \mathrm{G}$ 703 4, 3G 739 5, 3G 741 5, 2H 828 e 3H 843 foram identificados com características de tolerância ao déficit hídrico.

\section{Referências}

ALLEN, R. G.; PEREIRA, L. S.; RAES, D.; SMITH, M. Crop evapotranspiration: guidelines for computing crop water requirements. Roma:
FAO, 1998. 328 p. (FAO Irrigation and Drainage Papers, 56).

ARAUS, J. L.; SLAFER, G. A.; ROYO, C. SERRET, M. D. Breeding for yield potential and stress adaption in cereals. Critical Review in Plant Science, Boca Raton, v. 27, p. 377-412, 2008. http://dx.doi.org/10.1080/07352680802467736

BAI, L. P.; SUI, F. G.; GE, T. D.; SUN, Z. H.; LU, Y. Y.; ZHOU, G. S. Effect of soil drought stress on leaf water status, membrane permeability and enzymatic antioxidant system of maize. Pedosphere, Beijing v. 6, n. 3, p. 326-332, 2006.

BASTOS, E. A.; CARDOSO, M. J.; SOUSA, R. S. de; ANDRADE JUNIOR, A. S. de; RIBEIRO, V. Q.; BRITO, R. R. de. Productive performance of maize genotypes under water deficit in Teresina, Piauí State, Brazil. In: INTERNATIONAL CONFERENCE OF AGRICULTURAL ENGINEERING, 2012, Valencia. Agriculture $\&$ engineering for a healthier life: abstracts. 
TABELA 3. Valores médios da eficiência de uso da água (EUA) de genótipos de milho submetidos a condições de irrigação plena (IP) e deficiência hídrica (DH) do estádio VT ao R2. Teresina, PI, 2010.

\begin{tabular}{|c|c|c|}
\hline \multirow{2}{*}{ Genótipo $^{1}$} & \multicolumn{2}{|c|}{$\operatorname{EUA}\left(\mathrm{kg} \mathrm{ha}^{-1} \mathrm{~mm}^{-1}\right)$} \\
\hline & DH & IP \\
\hline AG 7088 & $17,14 \mathrm{~A}$ & $15,93 \mathrm{~A}$ \\
\hline $3 \mathrm{G} 7335$ & $9,85 \mathrm{~B}$ & $9,62 \mathrm{E}$ \\
\hline $1 \mathrm{G} 7034$ & $9,60 \mathrm{~B}$ & $12,30 \mathrm{C}$ \\
\hline $3 \mathrm{G} 7395$ & $8,89 \mathrm{~B}$ & $9,69 \mathrm{E}$ \\
\hline $3 \mathrm{G} 7415$ & $8,47 \mathrm{~B}$ & $10,31 \mathrm{D}$ \\
\hline $2 \mathrm{H} 828$ & 7,66 C & $7,86 \mathrm{E}$ \\
\hline $3 \mathrm{H} 843$ & $7,65 \mathrm{C}$ & $9,23 \mathrm{E}$ \\
\hline $2 \mathrm{H} 834$ & $7,20 \mathrm{C}$ & $13,42 \mathrm{~B}$ \\
\hline 1H 795 & 7,12 C & $11,92 \mathrm{C}$ \\
\hline 1D 2255 & $6,93 \mathrm{C}$ & $12,05 \mathrm{C}$ \\
\hline 1F 5834 & $6,83 \mathrm{C}$ & $9,24 \mathrm{E}$ \\
\hline 1F 5574 & $6,66 \mathrm{C}$ & $10,75 \mathrm{D}$ \\
\hline 1F 6325 & $6,22 \mathrm{D}$ & $11,85 \mathrm{C}$ \\
\hline $1 \mathrm{G} 6724$ & $6,20 \mathrm{D}$ & $10,59 \mathrm{D}$ \\
\hline $3 \mathrm{~F} 6245$ & $6,14 \mathrm{D}$ & $11,96 \mathrm{C}$ \\
\hline $3 \mathrm{H} 823$ & $6,13 \mathrm{D}$ & $10,30 \mathrm{D}$ \\
\hline 1H 845 & $6,08 \mathrm{D}$ & $9,18 \mathrm{E}$ \\
\hline $3 \mathrm{H} 832$ & $6,04 \mathrm{D}$ & $9,86 \mathrm{D}$ \\
\hline 3Н 798 & $5,87 \mathrm{D}$ & $8,89 \mathrm{E}$ \\
\hline 2B 707 & $5,69 \mathrm{D}$ & $10,80 \mathrm{D}$ \\
\hline $2 \mathrm{H} 829$ & $5,52 \mathrm{D}$ & $10,97 \mathrm{D}$ \\
\hline 3Н 813 & 4,92 E & $7,71 \mathrm{E}$ \\
\hline $3 \mathrm{G} 7385$ & $4,80 \mathrm{E}$ & $12,15 \mathrm{C}$ \\
\hline $3 \mathrm{H} 842$ & $4,61 \mathrm{E}$ & $11,20 \mathrm{C}$ \\
\hline $3 \mathrm{E} 4824$ & $4,60 \mathrm{E}$ & $10,84 \mathrm{D}$ \\
\hline $2 \mathrm{H} 826$ & $4,46 \mathrm{E}$ & $10,21 \mathrm{D}$ \\
\hline
\end{tabular}




\begin{tabular}{ccc}
$2 \mathrm{H} 831$ & $4,22 \mathrm{E}$ & $9,19 \mathrm{E}$ \\
$30 \mathrm{~F} 35$ & $4,21 \mathrm{E}$ & $14,23 \mathrm{~B}$ \\
$2 \mathrm{E} 5305$ & $4,16 \mathrm{E}$ & $11,55 \mathrm{C}$ \\
$1 \mathrm{~F} 6265$ & $4,06 \mathrm{E}$ & $9,54 \mathrm{E}$ \\
BRS 1040 & $3,99 \mathrm{E}$ & $8,48 \mathrm{E}$ \\
3F 6275 & $3,90 \mathrm{E}$ & $11,12 \mathrm{C}$ \\
1D 2195 & $3,74 \mathrm{E}$ & $8,92 \mathrm{E}$ \\
1F 5924 & $3,20 \mathrm{~F}$ & $9,70 \mathrm{E}$ \\
1H 787 & $2,88 \mathrm{~F}$ & $11,98 \mathrm{C}$ \\
1F 6255 & $2,86 \mathrm{~F}$ & $9,24 \mathrm{E}$ \\
\hline Média & 6,07 & 10,63 \\
CV $(\%)$ & 14,33 & 10,52 \\
Teste F & $* *$ & $* *$ \\
\hline
\end{tabular}

${ }^{\top}$ Médias seguidas da mesma letra, maiúscula na coluna, não diferem entre si pelo teste de Scott-Knott a 5\% de probabilidade; **Significativo ao nível de $1 \%$ de probabilidade pelo teste $\mathrm{F}$.

Valencia: CIGR: EurAgEng, 2012. Disponível em: http://ainfo.cnptia.embrapa.br/digital/ bitstream/item/65630/1/Productive10610001. pdf. Acesso em: 29 out. 2013.

BASTOS, E. A.; ANDRADE JÚNIOR, A. S. Boletim Agrometeorológico do ano de 2008 para o município de Teresina, PI. Teresina: Embrapa Meio-Norte, 2008, 37 p. (Embrapa MeioNorte. Documentos, 181).

BEIRAGI, M. A.; EBRAHIMI, M.; MOSTAFAVI, K.; GOLBASHY, M.; KHORASANI, S. K. A study of morphological basis of corn (Zea mays L.) yield under drought stress condition using correlation and path coefficient analysis.

Journal of Cereals and Oilseeds, Lagos, v. 2, n. 2, p. 32-37, 2011.

BERGAMASCHI, H.; DALMAGO, G. A.; BERGONCI, J. I.; BIANCHI, C. A. M.; MÜLLER, A. G.; COMIRAN, F.; HECKLER,
B. M. M. Distribuição hídrica no período crítico do milho e produção de grãos. Pesquisa Agropecuária Brasileira, Brasilia, DF, v. 39, n. 9, p. 831-839, 2004.

BRUCE, W. B.; EDMEADES, G. O.; BARKER, T. C. Molecular and physiological approaches to maize improvement for drought tolerance. Journal of Experimental Botany, London, v. 53, n. 66, p. 13-25, 2002.

ÇAKIR, R. Effect of water stress at different development stages on vegetative and reproductive growth of corn. Field Crops Research, Amsterdam, v. 89, p. 1-6, 2004.

CARDOSO, M. J.; BASTOS, E. A.; RIBEIRO, V. Q.; GUIMARÃES, L. J. M.; GUIMARÃES, P. E. O.; ROCHA, L. M. P. Performance produtiva e eficiência de uso da água de híbridos de milho em condições hídricas contrastantes. In: SIMPÓSIO DE MUDANÇAS CLIMÁTICAS 
E DESERTIFICAÇÃO NO SEMIÁRIDO BRASILEIRO, 3., 2011, Juazeiro. Experiências para mitigação e adaptação Anais... Petrolina: Embrapa Semiárido, 2011. 1 CD ROM.

CARDOSO, M. J.; BASTOS, E. A.; PACHECO, C. A. P.; ROCHA, L. M. P.; GUIMARÃES, L. J. M.; GUIMARÃES, P. E. DE O.; PARENTONI, S. N.; SILVA, A. R. Rendimento de Grãos e Componentes de Rendimento de Híbridos Comerciais de Milho sob Deficiência Hídrica. In: CONGRESSO NACIONAL DE MILHO E SORGO, 28.; ; SIMPÓSIO BRASILEIRO SOBRE LAGARTA DO CARTUCHO, 4., 2010, Goiânia. 2010, Potencialidades, desafios

e sustentabilidade: resumos expandidos. Goiânia: ABMS, 2010. 1 CD ROM.

CONAB. Acompanhamento de safra brasileira: grãos, décimo segundo levantamento. Brasília, DF, 2013. 29 p. Disponível em: http://www.conab.gov.br/OlalaCMS/uploads/ arquivos/13_10_16_14_32_01_boletim portugues_-_setembro_2013.pdf. Acesso em: 25 out. 2013.

CRUZ, D. D. Programa Genes: Biometria. Ed. UFV. Viçosa (MG), 2006. 382p.

DOORENBOS, J.; KASSAM, A. H. Efeito da água no rendimento das culturas. Campina Grande: UFPB, 1994. 306 p. (Estudos FAO: Irrigação e Drenagem, 33).

ClAESSEN, M. E. C. (Org.). Manual de métodos de análises de solos. 2. ed. rev. atual. Rio de Janeiro: EMBRAPA-CNPS, 1997. 212 p. (EMBRAPA-CNPS. Documentos, 1).
GOMIDE, R. L.; DURÃES, F. O. M.; GUIMARÃES, C. M.;ANDRADE, C. L. T.; ALBUQUERQUE, P. E. P.; BASTOS, E. A.; VIANA, J. H. M.; STONE, L. F.; MORAIS, O. P.; DEL PELOSO, M. J.; MAGAlHÃES, P. C.; MORGADO, L. B.; OLIVEIRA, A. C. Drought tolerance phenotyping in crops under contrasting target environments: procedures and practices. In: MONNEVEUX, P.; RIBAUT, J. M. (Ed). Drought phenotyping in crops: from theory to practice. México: CGIAR: CIMMYT, 2011. pt. 1, p. 51-91.

MAGALHÃES, P. C.; SOUZA, T. C.; ALBUQUERQUE, P. E. P.; KARAM, D.; MAGAlHÃES, M. M.; CANTÃO, F. R. O. Caracterização ecofisiológica de linhagens de milho submetidas a baixa disponibilidade hídrica durante o florescimento. Revista Brasileira de Milho e Sorgo, Sete Lagoas, v. 8, n. 3, p. 223-232, 2009.

PIMENTEL GOMES, F. Curso de estatística experimental. 13. ed. São Paulo: Nobel, 1990. $467 \mathrm{p}$.

RITCHIE, S. W.; HANWAY, J.; BENSON, J. B. How a corn plant develops. Ames: Iowa State University of Science and Technology, 1993. 26 p. (Special Report, 48).

SAS INSTITUTE. SAS/STAT: user's guide. 4. ed. v. 6. Cary, 1989. 943 p.

TAIZ, L.; ZEIGER, E. Fisiologia Vegetal. 4. ed. Porto Alegre: Artmed, 2009. 819 p.

ZIMMERMANN, F. J. P. Estatística aplicada à pesquisa agrícola. Santo Antônio de Goiás: Embrapa Arroz e Feijão, 2004. 402 p. 